\title{
A PARADIGM FOR INVESTIGATING THE EFFECTS OF TOP-DOWN PROCESSES ON VISUAL SEARCH
}

\author{
Arnab Biswas ${ }^{1}$, Devpriya Kumar ${ }^{2}$ \\ ${ }^{1}$ Department of Biological Sciences and Bioengineering \\ ${ }^{2}$ Department of Humanities and Social Science \\ ${ }^{2}$ Cognitive Science Program \\ Indian Institute of Technology, Kanpur
}

India

Please direct correspondence to arnabbiswas@ntu.edu.sg 


\begin{abstract}
Searching for things is an essential part of our everyday life. The way we search gives us clues on how our cognitive processes function. Scientists have used the visual search task to study attention, perception, and memory. Visual search performance depends upon a combination of stimulus-driven, bottom-up information, goal-oriented, top-down information, and selection history bias. It is difficult to separate these factors due to their close interaction. Our current study presents a paradigm to isolate the effects of top-down factors in visual search. In our experiments, we asked subjects to perform two different search tasks. A part of the total trials in each of these tasks had the same bottom-up information. That is, they had the same target, distractor, and target-distractor arrangement. We controlled for selection history bias by having an equivalent proportion of target types for all tasks and randomized the trial-order for each subject. We compared the mean response times for the critical trials, which had identical bottom-up information shared across the two pairs of tasks. The results showed a significant difference in mean response times of critical trials for both our experiments. Thus, this paradigm allows us to compare the difference in top-down guidance when controlling for bottom-up factors. Pairwise comparison of top-down guidance for different features given the same bottom-up information allows us to ask interesting questions such as, "Visual search guidance for which features can or cannot be easily increased by top-down processes?" Answers to these questions can further shed light on the ecological and evolutionary importance of such features in perception.
\end{abstract}

Index Terms—visual search, top-down processing, guided search, attention, perception 


\section{Introduction}

Every day, we come across instances where we need to search for things. Maybe you need to search for your keys on the desk, look out for your friend in the crowd, or search for apples in the supermarket aisle. More often than not, we end up finding what we search for. Sometimes the search is easy. For example, if your keys have a large red keychain, then they are easy to spot on the desk. At other times the search is difficult, like in a coffee shop where you're meeting your friend. Finding them may be difficult because of how crowded the place is or the uncertainty of whether your friend is already there.

Why are some searches harder than others? What determines visual search efficiency? The factors responsible for guiding visual search can be narrowed down to three main elements (Awh, Belopolsky \& Theeuwes, 2012; Wolfe \& Horowitz, 2017). The first element is stimulus-driven, saliency-based guidance of attention also called bottom-up guidance. Due to the visual features which make up a scene, bottom-up guidance directs our attention to a specific part of the scene over others. In the keychain example mentioned above, it is due to the fact that the keychain is big and red that makes it more noticeable compared to other items on your desk, and thus it is easily spotted. The second element is goal-oriented, user-driven guidance, or top-down guidance, in which attention is directed to parts of the scene having similar properties to the targeted item. For example, in the coffee shop example above, if you know that your friend is wearing a particular hat, then it is easier for you to constrain your search towards people who are wearing similar hats. And the last element is our selection history where our attention is biased by our past experiences of visual search. Basically, we can be primed for the next search based on the contents of all our prior selections. Take the example of a lost spoon, it is highly likely you will restrict your search to the kitchen or places it is highly likely to be based on your past experiences. If 


\section{EFFECT OF TOP-DOWN PROCESSES ON VISUAL SEARCH}

someone places the spoon in your wardrobe, a place you are not expecting it to be, you are going to have a hard time searching for it.

Many theories have tried to explain search performance based on bottom-up factors and how they are processed. Triesman and Gelade (1980) suggested that search efficiency depends on the number of features shared amongst the targets and distractors. If the targets and distractors are distinct along a basic feature dimension (e.g., color, shape, orientation, etc.; for a comprehensive list see Wolfe \& Horowitz 2004; Wolfe, 2014), then the search is relatively easy, such that the target usually 'pops-out' in our field of view. On the other hand, if the targets and distractors are defined by two or more feature dimensions and the target is not distinct from distractors in each of the dimensions then attention is required to 'bind' the features and search for the target. This makes the search relatively difficult.

Other studies have shown that peripheral vision also plays a crucial role in search tasks (Erkelens \& Hooge, 1996; Geisler, Perry \& Najemnik, 2006). Our visual resolution decreases dramatically as we move away from the fovea, the center of our vision, to the periphery (Green, 1970). Despite this peripheral bottleneck, sometimes the object we are searching for 'pops-out' in our periphery, making the search easier. At other times we need to make multiple rapid eye movements, to foveate onto objects before we find what we are looking for. This suggests that it is easier to search for objects that are unaffected by this peripheral bottleneck (Rosenholtz, Huang, Raj, Balas, \& Ilie 2012).

Search performance is also affected by top-down effects when we are driven by the goal to search for specific objects in memory. In fact, when searching in the real world, top-down processes dominate guidance (Chen \& Zelinsky, 2006). The guided search model (Wolfe, 1994; Wolfe \& Gray, 2007) suggests that when searching for a particular object, our attention boosts regions in the scene likely to contain similar features as the target. Thus, the 
search can be 'guided' by our top-down processes, resulting in quicker reaction time than a random search for the target across a scene where the targets and distractors share features.

Numerous studies have looked at the question of whether bottom-up or top-down control of attention dominates visual search (Egeth \& Yantis, 1997; Theeuwes, 1993, 1994; Theeuwes, Reimann, \& Mortier, 2006; Wolfe, Butcher, Lee, \& Hyle, 2003). Studies have shown that top-down and bottom-up processes are independent (Pinto, van der Leij, Sligte, Lamme \& Scholte 2013). But because of the automaticity of bottom-up processes, it is difficult to isolate it from top-down processes. Eye-tracking while free-viewing an image gives us a reasonable estimate of how bottom-up processes alone affect search. However, there have been no studies investigating how top-down processes affect search that is independent of bottom-up information. In this study, we try to address this. We hypothesized that if we controlled for bottom-up effects and selection history across two different search tasks, we will be able to determine the effect of top-down processes on search efficiency.

We conducted two experiments. Each experiment consisted of two visual search tasks: Task A and Task B. In each of these tasks, the target could be either one of the two objects denoted as possible targets and the set of distractors was constituted from the two predetermined distractors. Among all the various possible permutations of target and distractors across both tasks (target-present condition), we identified three different types- 1) where the target can be identified based on Task A only, 2) where target can be identified based on Task B only, and 3) where target can be identified based on either Task A or Task B. This third type of permutation has the same target and distractors. Further, controlling for the target-distractor arrangement resulted in trials with the same bottom-up information between the two tasks. We denoted these identical trials as critical trials (CTs). Comparing 
the response times for CTs across the two tasks in both experiments gave us a measure of the effect of top-down processes on visual search reaction times.

\section{EXPERIMENT 1}

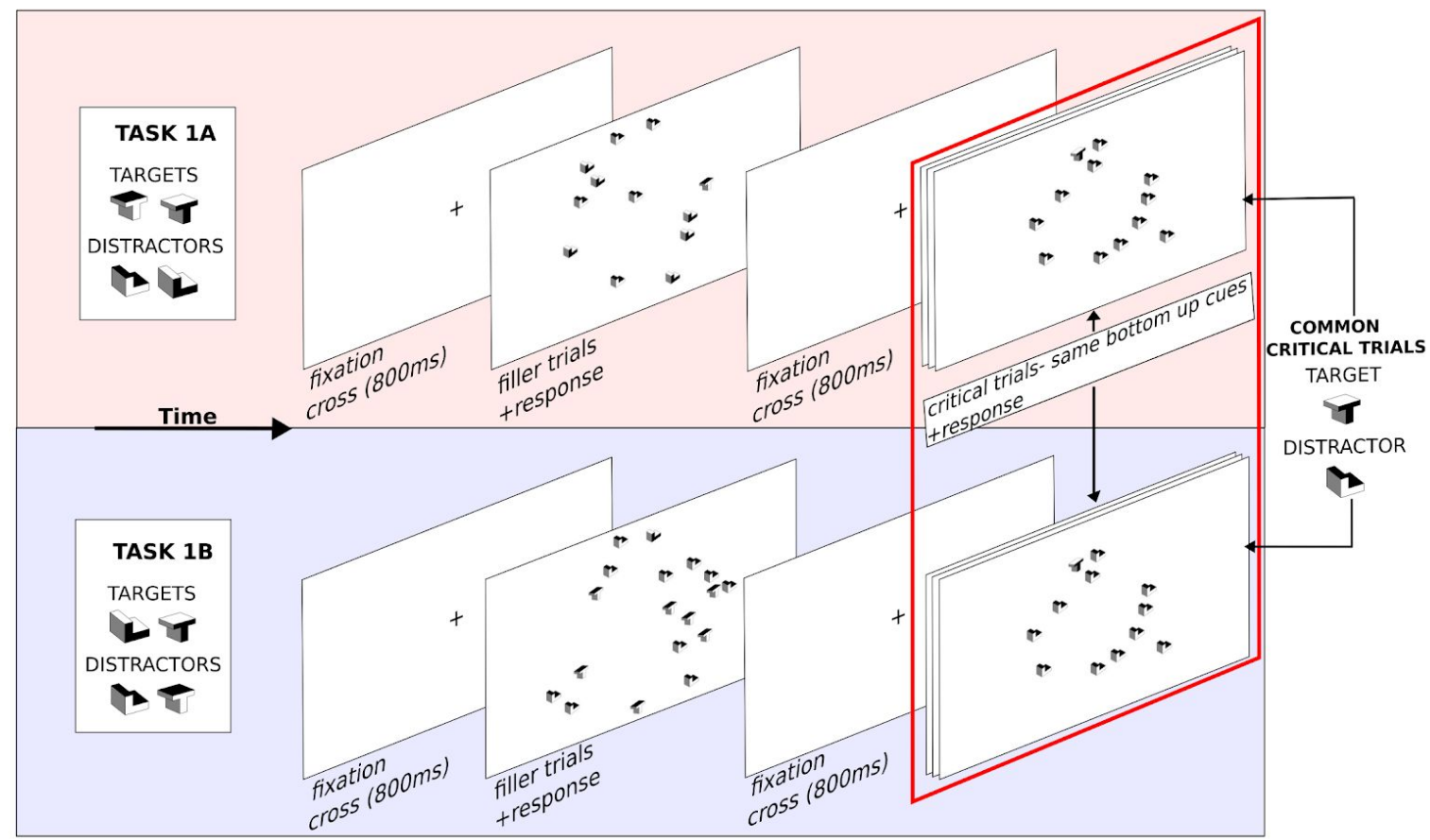

Figure 1. Experiment 1 Stimuli and Procedure

Experiment 1 consisted of two search tasks. Task $1 \mathrm{~A}$ required subjects to search for $T s$ amongst $L s$ and Task $2 B$ required subjects to search for top-lit $T$ s and $L$ s from bottom-lit $T s$ and Ls. Both tasks had a set of common trials that required subjects to search for a top-lit $T$ from amongst bottom-lit Ls. 


\section{EXPERIMENT 2}

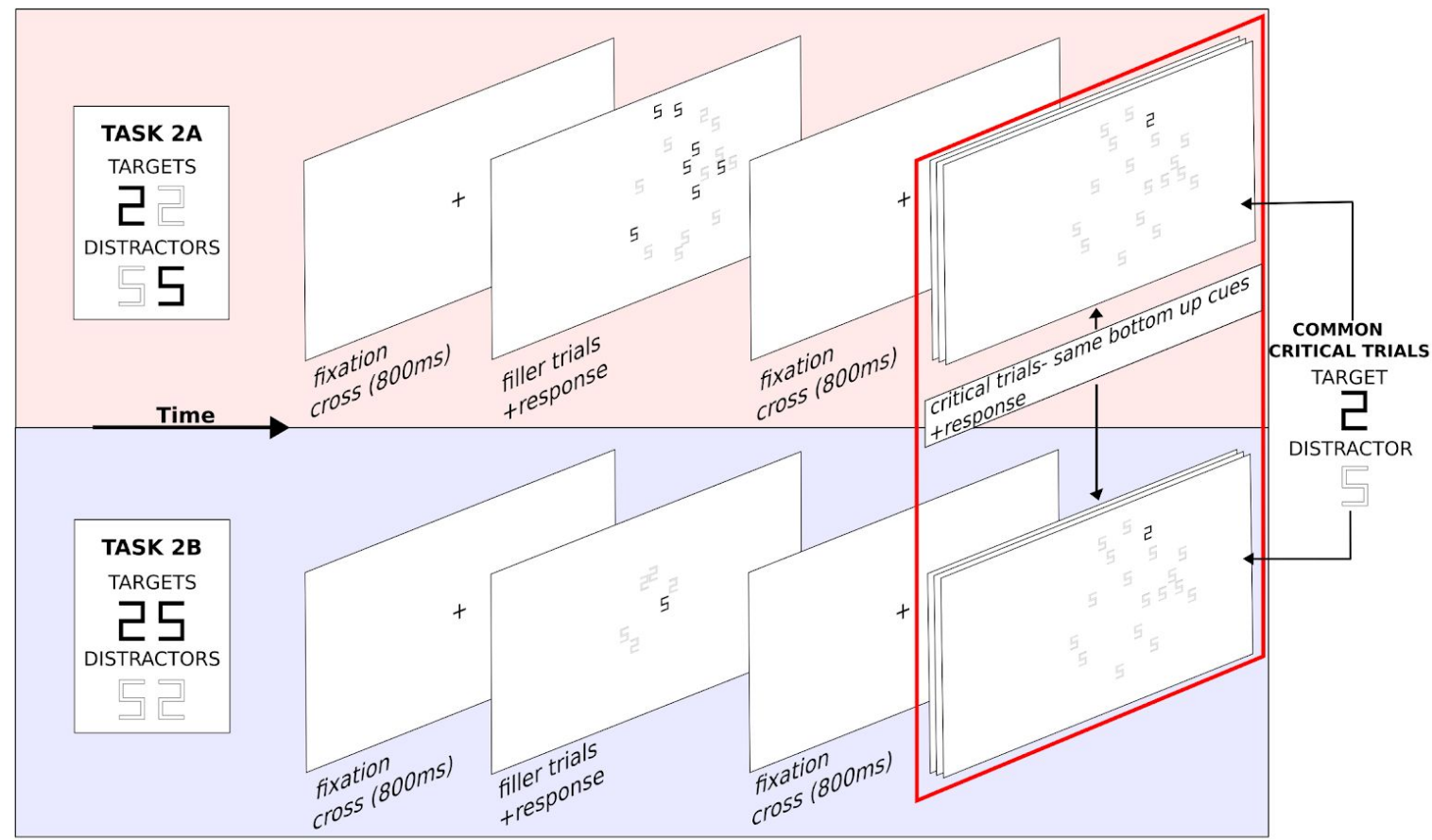

Figure 2. Experiment 2 Stimuli and Procedure

Experiment 2 consisted of two search tasks. Task $2 A$ required subjects to search for black or white $2 s$ amongst black or white 5 s and Task $2 B$ required subjects to search for black $2 s$ or $5 s$ from white $2 s$ and 5 s. Both tasks had a set of common trials that required subjects to search for a black 2 from amongst 5 s.

\section{Methods}

\section{Participants}

We recruited students from our university to participate in the experiment over two sessions. Ten subjects (three females, age 19-27) participated in Experiment 1, and we recruited ten more (four females, age 21-24) for completing Experiment 2. All subjects had normal or 
corrected to normal vision, were reimbursed appropriately and had given their consent to participate in the experiment.

\section{Stimuli \& Apparatus}

We had two types of stimuli based on the experiment. Experiment 1 consisted of search arrays made of the three-dimensional letters 'T's and 'L's (see Figure 1) which were either bottom-lit or top-lit, defined so based on the front face color of the object being black (top-lit) or white (bottom-lit). Each of the three colored faces (i.e., white, grey, and black) of the targets and distractors had an equal number of pixels to prevent search guidance by color. For Experiment 2, the search array was made up of the numerals 2 and 5 (see Figure 2), which were either black or white in color. All search arrays had a set size of $1,12,16$, or 18 . Each target and distractor subtended a visual angle of 1 degree. Target and distractors were randomly placed with added positional jitter (up to 1/8th degree) on four concentric circles having radii $4,5.5,7$, and 8.5 degrees of visual angle. We conducted the experiment in a dark room with stimuli presented on a 21 -inch LCD monitor to subjects seated at a distance of $70 \mathrm{~cm}$ from the screen. We used the Psychophysics toolbox 3 (Brainard, 1997; Pelli, 1997; Kleiner, Brainard, \& Pelli, 2007) in MATLAB for stimulus creation, presentation, and recording the subject's response.

\section{Procedure}

Both experiments followed the same general procedure. Each visual search task consisted of 20 practice trials, followed by a total of 128 experimental trials. Out of these 128 trials, 32 did not have a target, and 48 were CTs.

We asked subjects to search for specified targets amongst the possible distractors as quickly and accurately as possible. At the beginning of the experiment, we presented the 
subjects with written instructions on the screen which described the task, along with a pictorial representation of the two possible targets and the possible distractors (see Figure 1 and 2). Before each trial, subjects were required to fixate on a cross displayed for $600 \mathrm{~ms}$. After this, the search array was displayed. As soon as a target was determined to be present, the subject pressed a designated key. If the target was determined to be not present, they pressed another designated key. We defined the response time of a trial as the time from when the search array was displayed to the time of key release. We recorded the response time for all trials. In the case of an incorrect response, the subject had to repeat the trial later at some random point.

In Experiment 1, Task 1A, the subjects were asked to search for a T amongst L's and in Task 1B, they were asked to search for top-lit (based on the front face color) L's or T's among bottom-lit L's or T's (see Figure 1). Across both these tasks, the CTs required the subjects to search for a top-lit T amongst bottom-lit L's.

Similarly, in Experiment 2, Task 2A, subjects were asked to search for the numeral 2 amongst 5 s and in Task 2B subjects searched for a black colored 2 or 5 among white colored 2s and $5 \mathrm{~s}$ (see Figure 2). CTs in the second experiment required subjects to search for a black colored 2 among white colored 5 s.

For both experiments, comparing the response time for CTs across both these tasks gave us a measure of how much top-down processes affected search efficiency when controlling for the bottom-up processes. All participants were oblivious to the CTs manipulation. Search tasks within each experiment were counterbalanced across subjects. Subjects were asked to take a 20-minute mandatory break between each search task. 


\section{Analysis}

For every subject, we first filtered the response time data for each task by discarding response outside the range of $+/-3$ SD for each set size. Filtering led to less than $1 \%$ of trials being discarded. Only target-present trials with a valid response were analyzed. The mean response time as a function of set size is a standard measure of search efficiency for visual search tasks. We used the same for our analysis.

We performed a repeated-measures ANOVA on the response time of CTs. In our ANOVA model, the response time was dependent on two factors- task condition (Task A or Task B within an experiment) and set size $(1,6,12$, or 18$)$. If the main effect was found, we performed a Bonferroni corrected pairwise t-test between conditions to determine significant effects. All analyses were performed using R (R Core Development Team, 2009). We used the package ggplot2 for making plots and sjstats for calculating the effect size.

\section{Results}

For each of the four tasks, we plotted the mean response times for all trials with target-present and valid responses for all subjects as a function of set size (see Figure 3). We also plotted the response time for just the CTs for all subjects. Based on our hypothesis, we expected to see a difference in mean response times for CTs in both experiments.

For Experiment 1 we found a main effect of task $F(1,9)=107.689, p<0.001, \eta^{2}=0.203$ and a main effect of set size $F(3,27)=74.835, p<0.01, \eta^{2}=0.382$. Bonferroni corrected pairwise t-tests for all the set sizes were significant $p<0.001$ except for the pair $12-18$. The interaction between task and set-size was also significant $F(3,27)=12.246, p<0.001, \eta^{2}=0.059$. Similarly for Experiment 2, we found a main effect of task $F(1,9)=40.279, p<0.001, \eta^{2}=0.368$ a main effect of set size $F(3,27)=12.863, p<0.001, \eta^{2}=0.074$. Bonferroni corrected pairwise t-tests 
for set size were not significant for set size pairs 6-12, 6-18 and 12-18. For Experiment 2, the interaction between task and set-size was significant $F(3,27)=3.499, p=0.029, \eta^{2}=0.017$.
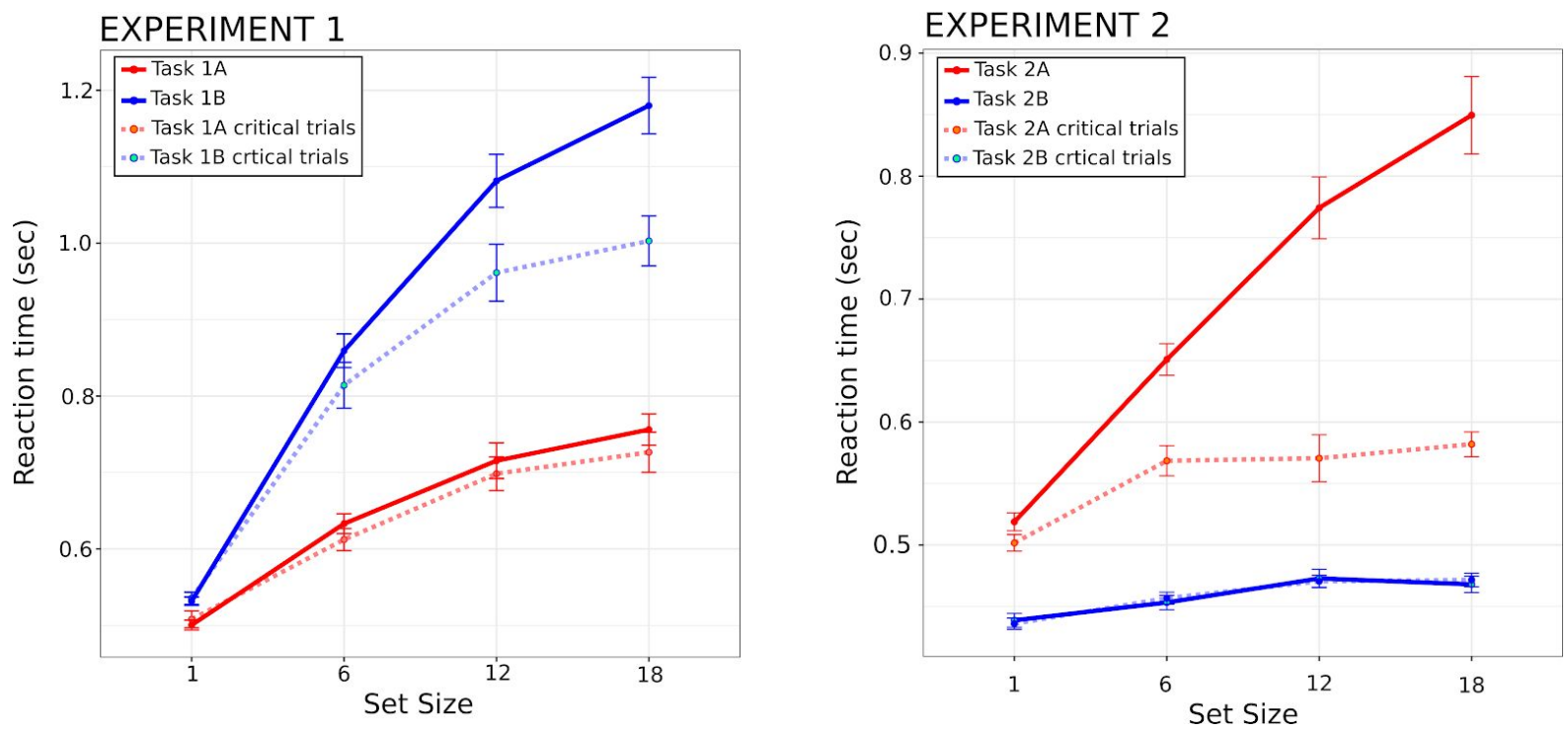

Figure 3. Mean response time plots as a function of set size for each experiment.

The mean response times for each task as a function of set size(solid lines) is a good estimator of search efficiency. Response times for critical trials have been plotted separately (dashed lines). The higher the slope of the curve the more difficult the task. The error bars denote the SEM.

\section{Discussion}

We conducted two experiments; each had two visual search tasks. $37.5 \%$ of the trials in both these tasks, termed critical trials (CTs), had identical bottom-up information. Further, we minimized the effect of selection history bias on search performance by randomizing trial order and using an equal proportion of the two possible targets. Looking at our results, we note a significant difference in mean reaction times for CTs in both experiments. Since we 
controlled for bottom-up effects and selection history bias, this difference in reaction times for CTs is purely due to top-down processes.

\section{Interpreting results based on the Guided Search model}

Below we interpret our results based on the Guided Search model (Wolfe, 1994; Wolfe \& Gray, 2007) as it is the most appropriate to consider the difference due to top-down guidance. The Guided Search model suggests that a location-dependent activation map of the visual input guides the deployment of attention. Greater activation of a region in the map means that the corresponding region in the scene attracts more attention relative to those with lower activation. According to the model, for a particular trial, we first generate feature based bottom-up activation maps. These feature activation maps describe the visual input in terms of the particular feature dimension. For example, the color feature map would contain all the information about the different colors present in the scene at every location and similarly for other feature maps such as orientation, shape, and others. Feature maps are generated in parallel for each basic feature and available to us pre-attentively. The more a location in a scene is different from the rest in a particular feature dimension, the higher its bottom-up activation. Next, top-down attention leads to further activation in the generated bottom-up feature maps. For example, if we were searching for a vertical and red target amongst horizontal and green distractors, the regions in the color feature map with red and the regions in the orientation feature map with vertical would have higher activation. The final activation map is simply a weighted sum of the bottom-up activation and the top-down activation of all the feature maps plus a noise variable. Attention is sequentially guided to regions in the scene in order of decreasing activation.

Now considering Experiment 1, Task $1 \mathrm{~A}$ is in essence, a shape search for Ts amongst Ls. The lighting direction is a confounding feature as the target $\mathrm{T}$ can be both top-lit or bottom-lit. 
We note that both shape (Bergen \& Julesz, 1983; Kristjansson \& Tse, 2001; Orsten-Hooge, Portillo, \& Pomerantz, 2015) and lighting- direction (Aks \& Enns, 1992; Kleffner \& Ramachandran, 1992; Sun \& Perona,1996; Zhang, Huang, Yigit-Elliott, \& Rosenholtz, 2015) are basic features which can pre-attentively guide our search. The activation map for Task $1 \mathrm{~A}$ would have a higher activation for regions with Ts and have no additional top-down activation for lighting-direction because the targets and distractors do not differ in this feature dimension. Now in Task 1B, subjects needed to search for top-lit Ts and Ls. In this case, there would be higher activation for top-lit items in the scene as compared to those which are bottom-lit and no additional activation based on shape. Comparing the activation for CTs across tasks, we note that there will be identical bottom-up activation, with any difference in activation being due to top-down processes. The mean response times for the critical trials in Task $1 \mathrm{~A}$ are shorter than Task 1B. Therefore, in this experiment, top-down activation for the shape feature can better guide search than lighting direction.

Similarly analyzing Experiment 2, we see Task 2A, as a search for black and white 2s among 5s. The targets and distractors differ in the shape feature dimension, with color being the confounding feature. Color (Carter, 1982; Daoutis, Pilling, \& Davies, 2006; Treisman \& Souther, 1985; Treisman \& Gormican, 1988;) is a basic feature just like, shape. In Task 2A, top-down activation would be higher for regions with $2 \mathrm{~s}$ in each trial, with no additional activation based on color. On the other hand, Task $2 \mathrm{~B}$ is a color search for black $2 \mathrm{~s}$ or $5 \mathrm{~s}$ amongst white $2 \mathrm{~s}$ or $5 \mathrm{~s}$. Here top-down activation would be higher for regions in a trial with black targets with no further activation due to shape. Now, because bottom-up activation across CTs is similar, looking at the response time plots, we can say that in Experiment 2 top-down activation for color is much higher than that for shape. 


\section{Ecological and evolutionary importance of features in perception}

There are many examples that suggest that evolution may have shaped the process by which we search for things. For instance, Wolfe, (2013) has shown that animal foraging models can be used to predict how humans search across multiple search scenes. Evolution might also explain why certain features easily guide visual search. For example, Zhang et al. (2015) state that light-direction can only guide the search for objects which are top-lit, as opposed to bottom- lit, because of the way we experience objects in the natural world. Another study shows that chimpanzees can search for upright faces faster among inverted faces (Tomonaga, 2007). Since faces are highly important for social behavior and we naturally experience mostly upright faces, it can be argued that preference for upright faces may be due to the way primates have evolved.

In the context of our experiment, we speculate that more weight is given to the bottom-up guidance due to certain features over others. These weights, which signify the importance of one feature over the other, may define the evolutionary importance of the feature. With features with higher weights possibly required for tasks related to threat detection, survival, or finding food. In our experiment, if for two competing feature maps the weighted bottom-up guidance for a feature is much more than the sum of the weighted bottom-up and top-down guidance for the other, then irrespective of the amount of top-down guidance, the feature with higher weighted bottom-up guidance will always attract more attention, even if that feature is irrelevant for a given task.

The paradigm used in this experiment can be used to determine the relative weights for two competing features. For example, in Experiment 2, despite the same bottom-up information, the top-down guidance due to color has resulted in a comparatively shorter reaction time than for the shape feature. Thus, we can argue that we assign a higher weight to bottom-up 
guidance due to color. This may reflect the evolutionary importance of prioritizing color over the shape. A full list of such features with their associated weights can give us clues on the ecological and evolutionary significance of highly weighted features for perception.

\section{Conclusion}

This paradigm provides an efficient method to determine the effect of top-down processes on visual search performance. It can also help us in answering crucial questions such as how search varies with a change in task description for the same bottom-up information? It is possible to use this paradigm along with eye-tracking to determine how our gaze patterns change due to different tasks for the same bottom-up cue. Eye-tracking can be further useful to determine if unimportant features for a particular task continue to guide attention despite the top-down activation of task-specific features. We speculate that we are hard-wired to pay attention to unimportant features and they cannot be ignored even if we consciously try to do so. A list of such features that can or cannot be easily ignored based on top-down activation can give us information about the ecological and evolutionary importance of such features in perception. In addition to this, other applications of this paradigm may include looking at the difference in $\mathrm{fMRI}$ activation across tasks for CTs to determine task-specific activation of regions in the brain.

\section{References}

Aks, D. J., \& Enns, J. T. (1992). Visual search for direction of shading is influenced by apparent depth. Perception \& Psychophysics, 52(1), 63-74.

Awh, E., Belopolsky, A. V., \& Theeuwes, J. (2012). Top-down versus bottom-up attentional 
control: A failed theoretical dichotomy. Trends in Cognitive Sciences, 16(8), 437-443.

Bergen, J. R., \& Julesz, B. (1983). Rapid discrimination of visual patterns. IEEE Transactions on Systems, Man, and Cybernetics, (5), 857-863.

Brainard, D. H. (1997). The psychophysics toolbox. Spatial Vision, 10, 433-436.

Carter, R. C. (1982). Visual search with color. Journal of Experimental Psychology: Human Perception and Performance, 8(1), 127.

Chen, X., \& Zelinsky, G. J. (2006). Real-world visual search is dominated by top-down guidance. Vision Research, 46(24), 4118-4133.

Daoutis, C. A., Pilling, M., \& Davies, I. R. (2006). Categorical effects in visual search for colour. Visual Cognition, 14(2), 217-240.

Egeth, H. E., \& Yantis, S. (1997). Visual attention: Control, representation, and time course. Annual Review of Psychology, 48(1), 269-297.

Erkelens, C. J., \& Hooge, I. T. C. (1996). The role of peripheral vision in visual search. Journal of Videology, 1, 1-8.

Green, D. G. (1970). Regional variations in the visual acuity for interference fringes on the retina. The Journal of Physiology, 207(2), 351-356.

Geisler, W. S., Perry, J. S., \& Najemnik, J. (2006). Visual search: The role of peripheral information measured using gaze-contingent displays. Journal of Vision, 6(9), 1-1.

Kleffner, D. A., \& Ramachandran, V. S. (1992). On the perception of shape from shading. Perception \& Psychophysics, 52(1), 18-36.

Kleiner, M., Brainard, D., \& Pelli, D. (2007), What's new in Psychtoolbox-3? Perception, 36 ECVP Abstract Supplement. 
EFFECT OF TOP-DOWN PROCESSES ON VISUAL SEARCH

Kristjánsson, Á., \& Tse, P. U. (2001). Curvature discontinuities are cues for rapid shape analysis. Perception \& Psychophysics, 63(3), 390-403.

Orsten-Hooge, K. D., Portillo, M. C., \& Pomerantz, J. R. (2015). False pop out. Journal of Experimental Psychology: Human Perception and Performance, 41(6), 1623.

Pinto, Y., van der Leij, A. R., Sligte, I. G., Lamme, V. A., \& Scholte, H. S. (2013). Bottom-up and top-down attention are independent. Journal of Vision, 13(3), 16-16.

Pelli, D. G. (1997). The VideoToolbox software for visual psychophysics: Transforming numbers into movies. Spatial Vision, 10, 437-442.

Rosenholtz, R., Huang, J., Raj, A., Balas, B. J., \& Ilie, L. (2012). A summary statistic representation in peripheral vision explains visual search. Journal of Vision, 12(4), 14-14.

R Core Development Team, (2013). R: A language and environment for statistical computing.

Sun, J., \& Perona, P. (1998). Where is the sun?. Nature Neuroscience, 1(3), 183.

Tomonaga, M. (2007). Visual search for orientation of faces by a chimpanzee (Pan troglodytes): face-specific upright superiority and the role of facial configural properties. Primates, 48(1), 1-12.

Theeuwes, J. (1993). Visual selective attention: A theoretical analysis. Acta Psychologica, 83(2), 93-154.

Theeuwes, J. (1994). Stimulus-driven capture and attentional set: selective search for color and visual abrupt onsets. Journal of Experimental Psychology: Human Perception and Performance, 20(4), 799. 
Theeuwes, J., Reimann, B., \& Mortier, K. (2006). Visual search for featural singletons: No top-down modulation, only bottom-up priming. Visual Cognition, 14(4-8), 466-489.

Treisman, A. M., \& Gelade, G. (1980). A feature-integration theory of attention. Cognitive Psychology, 12(1), 97-136.

Treisman, A., \& Souther, J. (1985). Search asymmetry: A diagnostic for preattentive processing of separable features. Journal of Experimental Psychology: General, 114(3), 285.

Treisman, A., \& Gormican, S. (1988). Feature analysis in early vision: evidence from search asymmetries. Psychological Review, 95(1), 15.

Wolfe, J. M. (1994). Guided search 2.0 a revised model of visual search. Psychonomic Bulletin \& Review, 1(2), 202-238.

Wolfe, J. M., Butcher, S. J., Lee, C., \& Hyle, M. (2003). Changing your mind: on the contributions of top-down and bottom-up guidance in visual search for feature singletons. Journal of Experimental Psychology: Human Perception and Performance, 29(2), 483.

Wolfe, J. M., \& Horowitz, T. S. (2004). What attributes guide the deployment of visual attention and how do they do it?. Nature Reviews Neuroscience, 5(6), 495.

Wolfe, J. M., \& Gray, W. (2007). Guided search 4.0. Integrated Models of Cognitive systems, 99-119.

Wolfe, J. M. (2013). When is it time to move to the next raspberry bush? Foraging rules in human visual search. Journal of Vision, 13(3), 10-10. 
EFFECT OF TOP-DOWN PROCESSES ON VISUAL SEARCH

Wolfe, J. M. (2014). Approaches to visual search: Feature integration theory and guided search. Oxford Handbook of Attention, 11-55.

Wolfe, J. M., \& Horowitz, T. S. (2017). Five factors that guide attention in visual search. Nature Human Behaviour, 1(3), 0058.

Zhang, X., Huang, J., Yigit-Elliott, S., \& Rosenholtz, R. (2015). Cube search, revisited. Journal of Vision, 15(3), 9-9. 